\title{
The dark side of paclitaxel
}

\author{
Alekha K. Dash
}

Published online: 27 May 2010

(C) Springer-Verlag 2010

The bark extract of Pacific Yew (Taxus brevifolia) was first reported to have anti-tumor activity in 1963. The active constituent responsible for this activity was later identified in 1971 as paclitaxel [1]. Paclitaxel is a cytotoxic drug used in the treatment of variety of tumors including ovarian, breast, and non-small-cell lung cancers [2].

Its unique mode of action relies on binding to microtubules, preferentially to $\beta$-tubulin. Paclitaxel interferes with the function of microtubules, either by destabilizing or stabilizing the microtubules. Paclitaxel stimulates phosphorylation of $\beta$-tubulin in both differentiated and undifferentiated P19 cells and stabilizes microtubules by the prevention of depolymerization which results in cell death [3]. In spite of its efficacy against wide varieties of tumors, it has some shortcomings.

Large scale production of this alkaloid from natural sources by extraction is a very challenging and expensive process. Only half a gram of paclitaxel can be obtained from a 40-foot tall Pacific Yew plant, it may take the plant 200 years to reach this height. The discovery of a four-step procedure that converts 10-deacetylbaccatin, a related compound found in various Yew species which grows significantly quicker than Pacific Yew, increased its yield and production [4].

Paclitaxel exhibits dose-limiting toxicity and a short half-life. After IV infusion, the initial and final elimination half-lives of paclitaxel were reported to be $0.29 \pm 0.13$ and $6.95 \pm 5.40 \mathrm{~h}$, respectively [5]. Ninety percent of the drug is hydroxylated by the CYPs 3A4, 3A5, 1A2 and CYP2C8

A. K. Dash ( $\bowtie)$

Department of Pharmacy Sciences,

School of Pharmacy and Health Professions,

Creighton University, Omaha, NE 68178, USA

e-mail: AlekhaDash@creighton.edu enzymes in the liver and excreted in feces [6]. Less than $10 \%$ of the drug is eliminated through the kidney, unaltered. The drug is bound up to $90 \%$ to tissue-protein [5].

Resistance to paclitaxel develops due to multidrug resistance-1 (MDR-1)/P-glycoprotein (PgP) expression, effect of drug efflux transporter (ABCB1), breast cancer resistance protein (BRCP), $\beta$-tubulin expression, over expression of oncogene c-erb-B-2, modification of apoptosis signaling sensitivity, or paclitaxel detoxification mediated by CYP [7]. Therefore, the required dose of paclitaxel increases with time, which in turn will cause greater toxicity.

Its physicochemical properties such as high molecular weight $(853 \mathrm{~g} / \mathrm{mol})$, lipophilicity ( $\log \mathrm{P}$ of 3.66 ) and poor aqueous solubility $(0.25 \mu \mathrm{g} / \mathrm{mL})$ exhibit critical formulation challenges. In order to overcome these challenges, a mixture of polyoxyethylated castor oil and anhydrous alcohol, commonly called Cremophor EL is used. The use of Cremophor EL has severe side effects that lead to hypersensitivity reaction, hyperlipidemia, and peripheral neuropathy. To avoid Cremophor EL in the formulation, various approaches have been investigated to enhance its bioavailability and reduce toxicity [8].

Over the last few years, extensive research has focused on identifying genetic and non-genetic factors responsible for inter-individual variability for paclitaxel pharmacokinetics, efficacy, and toxicity. Polymorphisms in genes encoding paclitaxel metabolizing enzymes, and presence of transporters have been considered as possible factors for this variability. Further studies of genes involved in the efficacy and elimination of paclitaxel has identified organic anion transporting polypeptide (OATP), and IB3. For example, IB3 is responsible for paclitaxel hepatic uptake, polymorphism in the genes encoding for paclitaxel metabolizing enzymes, and transporters such as CYP2C8, 
CYP3A4, CYP3A5, OATP1B3 and PgP [9]. However, the pharmacogenomic studies that have been reported so far have provided conflicting conclusions. For paclitaxel neurotoxicity risks, some studies have reported positive results for $\mathrm{ABCB} 1$ [10], CYP2C8, and CYP3A5 polymorphism, while others have found no such relation [11]. In the future, more research efforts are needed for the development of individual paclitaxel pharmacotherapy.

Conflict of interest None.

\section{References}

1. Wani MC, Taylor HL, Wall ME et al (1971) Plant antitumor agents: VI. The isolation and structure of taxol, a novel antileukemic and anti tumor agent from Taxus brevifolia. J Am Chem Soc 93:2325-2327

2. Eniu A, Palmieri FM, Perez EA (2005) Weekly administration of docetaxel and paclitaxel in metastatic or advanced breast cancer. Oncologist 10:665-685

3. Geney R, Sun L, Pera P et al (2005) Use of the tubulin bound paclitaxel conformation for structure-based rational drug design. Chem Biol 12:339-348
4. Holton RA, Kim HB, Somoza C et al (1994) First total synthesis of taxol: completion of the $\mathrm{C}$ and $\mathrm{D}$ rings. $\mathrm{J}$ Am Chem Soc 116:1599-1600

5. Rowinsky EK, Donehower RC (1995) Paclitaxel (taxol). N Engl J Med 332:1004-1014

6. Mutch E, Daly AK, Leathart JB et al (2003) Do multiple cytochrome P450 isoforms contribute to parathion metabolism in man? Arch Toxicol 77:313-320

7. Hopper-Borge E, Xu X, Shen T et al (2009) Human multidrug resistance protein 7 (ABCC10) is a resistance factor for nucleoside analogues and epothilone B. Cancer Res 69:178-184

8. Singh S, Dash AK (2009) Paclitaxel in cancer treatment: perspectives and prospects of its delivery challenges. Crit Rev Ther Drug Carrier Syst 26:333-372

9. Rodriguez-Antona C (2010) Pharmacogenomics of paclitaxel. Pharmacogenomics 11:621-623

10. Green H, Soderkvist P, Rosenberg P et al (2009) Pharmacogenetic studies of paclitaxel in the treatment of ovarian cancer. Basic Clin Pharmacol Toxicol 104:130-137

11. Marsh S, Paul J, King CR et al (2007) Pharmacogenetic assessment of toxicity and outcome after platinum taxane chemotherapy in ovarian cancer: the Scottish randomized trial in ovarian cancer. J Clin Oncol 9:4528-4535 